\title{
Estado del arte de la investigación en acceso a los servicios de salud en Colombia, 2000-2013: revisión sistemática crítica*
}

\section{State of the Art in Access to Health Services Research in Colombia, 2000-2013: a Systematic Review}

\section{Estado da arte da pesquisa em acesso aos serviços de saúde na Colômbia, 2000-2013: revisão sistemática crítica}

Fecha de recepción: 02-05-14 Fecha de aceptación: 23-06-14 Disponible en línea: 01-07-14 doi: 10.11144/Javeriana.rgyps13-27.eaia

Cómo citar este artículo:

Tovar-Cuevas LM, Arrivillaga-Quintero M. Estado del arte de la investigación en acceso a los servicios de salud en Colombia, 2000-2013: revisión sistemática crítica. Rev. Gerenc. Polít. Salud. 2014; 13(27):

12-26. http://dx.doi.org/10.11144/Javeriana.rgyps13-27.eaia

\author{
Luis Miguel Tovar-Cuevas** \\ Marcela Arrivillaga-Quintero***
}

Artículo de investigación, proyecto de investigación: Evaluación de acceso a servicios de salud y su relación con las inequidades sociales en el Pacífico colombiano, inicio: enero del 2013, finalización: diciembre del 2013, financiado por la Pontificia Universidad Javeriana-Cali (Colombia).

** M.Sc. Salud Pública, economista, profesor asistente del Departamento de Economía de la Facultad de Ciencias Económicas y Administrativas de la Pontificia Universidad Javeriana-Cali (Colombia), investigador del grupo de investigación Salud y Calidad de Vida, adscrito a dicha institución. Correo electrónico: Itovar@javerianacali.edu.co

*** Ph.D. Salud Pública, profesora asociada del Departamento de Salud Pública y Epidemiología de la Facultad de Ciencias de la Salud de la Pontificia Universidad Javeriana-Cali (Colombia), investigadora del grupo de investigación Desarrollo Regional, adscrito a dicha institución. Correo electrónico: marceq@javerianacali.edu.co 


\section{Resumen}

Este estudio presenta una revisión sistemática de los trabajos de investigación en el tema del acceso a servicios de salud en Colombia en el periodo 2000-2013. Las bases de datos empleadas para la búsqueda fueron Ebsco, Jstor, Proquest, ISI Web of Science, scielo y Cochrane. Los resultados mostraron que la mayoría de los estudios se efectuaron entre 2006 y 2011, la mayor parte de los cuales analizaron áreas urbanas. Una tercera parte consistió en una investigación sobre datos nacionales, en donde las temáticas estuvieron centradas en equidad en la utilización de servicios, algunos grupos de población vulnerables, acceso a algunos servicios específicos, así como en las implicaciones de la Ley 100 de 1993 en el acceso. Los resultados sugirieron que es necesario que Colombia mejore las fuentes de información, que los marcos teóricos sean adaptados al contexto y que se realicen estudios que aborden de manera integral todos los eslabones que involucran el acceso.

Palabras clave: accesibilidad a los servicios de salud; equidad en el acceso; revisión; Colombia; acceso a los servicios de salud; revisión sistemática

\section{Abstract}

This study shows a systematic review of research works on the topic of access to health services in Colombia in the period 2000-2013. The databases used were Ebsco, Jstor, Proquest, ISI Web of Science, scielo and Cochrane. Results showed that most of the studies were carried out between 2006 and 2011. The majority of them analyzed rural areas, and a third part consisted on analysis of national data. Thematically, they were focused on equality in the use of services, some vulnerable population groups, access to some specific services, as well as the implications of the law 100 of 1993 on access to health services. Results suggested that Colombian researchers need to improve their information sources, and that the theoretical frameworks must be adapted to the context. There is the need of more studies that address in a more comprehensive way all aspects involved in access.

Keywords: health services accessibility; equity in access; review; Colombia; access to health services; systematic review

\section{Resumo}

Este estudo apresenta revisão sistemática dos trabalhos de pesquisa no tema do acesso a serviços de saúde na Colômbia no período 2000-2013. As bases de dados empregadas para a procura foram Ebsco, Jstor, Proquest, ISI Web of Science, scielo e Cochrane. Os resultados mostraram que a maioria dos estudos foram realizados entre 2006 e 2011, a maior parte dos quais analisaram áreas urbanas. Uma terça parte consistiu em uma pesquisa sobre dados nacionais, na que as temáticas estiveram centradas em equidade na utilização de serviços, alguns grupos de população vulneráveis, acesso a alguns serviços específicos, assim como as implicações da Lei 100 de 1993 no acesso. Os resultados sugeriram que é preciso a Colômbia melhorar as fontes de informação, que os quadros teóricos forem adaptados ao contexto e que forem realizados estudos para abordar de maneira integral todos os elos que envolvem o acesso.

Palavras-chave: acessibilidade aos serviços de saúde; equidade no acesso; revisão; Colômbia; acesso aos serviços de saúde; revisão sistemática 


\section{INTRODUCCIÓN}

El acceso a los servicios de salud es un concepto que ha intentado explicar, en términos operativos, el funcionamiento en la puerta de entrada a los sistemas de salud, sea cual fuere su naturaleza (pública, privada o mixta), así como la relación entre pacientes que demandan atención en salud y diferentes servicios que componen tales estructuras sanitarias. A su vez, el acceso ha sido un constructo objeto de amplia investigación empírica en diversos escenarios a nivel global. Es así como la literatura científica muestra abundante evidencia sobre las características asociadas al acceso tanto en países y regiones de mayor y menor riqueza, como en aquellos con buenos y pobres resultados de salud.

Desde el punto de vista conceptual, la mayoría de los modelos empleados para abordar el problema del acceso se fundamentan en la lógica de mercado, e incluyen factores y dimensiones asociados a la oferta y a la demanda de servicios de salud (1-5), con especial énfasis en la atención médica. Estos modelos han servido como marcos de referencia conceptual en la mayor parte de las investigaciones realizadas en todos los contextos.

En Colombia, la información institucional oficial sobre acceso a servicios de salud es limitada y se restringe a cifras de cobertura de aseguramiento y a los indicadores de camas hospitalarias por 1000 habitantes, tasa de médicos por 10000 habitantes, número de consultas urgentes por cada 10 externas en la red pública, número de tutelas por no prestación de servicios del Plan Obligatorio de Salud (POS) o Plan Obligatorio de Salud Subsidiado (POS-S), porcentaje de partos con atención institucional, tasa de atención profesional recibida frente a una necesidad de salud, porcentaje de personas que enfrentan barreras de acceso a servicios de salud y barreras de acceso a los servicios de salud según causa (6).
El Ministerio de Salud solo reporta datos desagregados por departamento en cuanto a camas hospitalarias por 1000 habitantes y en número de consultas urgentes por cada 10 externas en la red pública; los datos de la red privada de prestación de servicios no están disponibles. Otros indicadores son observados por "Así Vamos en Salud", organización que sigue el sector salud en el país, mediante los indicadores de atención de partos en las instituciones de salud, coberturas de vacunación, de control prenatal y tratamientos entregados a pacientes con VIH/SIDA; solo la atención de partos institucionales cuenta con datos discriminados por departamento y el resto de los indicadores no tienen información desagregada disponible.

La base de datos única de afiliados del Ministerio de Salud, que aporta datos sobre el régimen de afiliación, muestra inequidades en el acceso entre regiones de Colombia y en el interior de ellas. Solo para citar un ejemplo, en la Región Pacífica del país, a abril del 2014, en el régimen subsidiado (para personas en situación de pobreza) había aproximadamente $88 \%$ de la población en el Chocó, $78 \%$ en el Cauca, $81 \%$ en Nariño y $44 \%$ en el Valle del Cauca; y en el régimen contributivo (para personas con capacidad de pago) $11 \%$ en el Chocó, $21 \%$ en el Cauca, $18 \%$ en Nariño y $55 \%$ en el Valle del Cauca (7). Al revisar los datos para los cuatro departamentos del Pacífico, se encuentran cifras preocupantes, particularmente en el Cauca, comparado con el resto de Colombia.

Para el 2008 (última información disponible consultada en el 2012), en este departamento había el menor número de camas por 1000 habitantes (solo 0,8 camas), junto con el departamento del Vaupés; en Chocó se reportaron 1,1 camas, en Nariño 1,4 y en Valle, 1,5; Quindío fue el departamento con más camas por 1000 habitantes, siendo de 2,1. En cuanto al número de consultas urgentes 
por cada 10 externas en la red pública, en el 2008 el Cauca tuvo 1,5, la más baja en todo el país; en Chocó 2,8 consultas, Nariño 2,4 y Valle 4,1; el mayor número de consultas fue de 5, en el departamento del Atlántico (6).

A la fecha de publicación de este artículo se tramita en el Congreso de la República otro intento de reforma al sistema de salud colombiano. Tras varios intentos previos, los problemas estructurales del sistema siguen sin resolverse y las barreras de acceso se han mantenido. Persiste la presencia de población sin cobertura en salud (concepto que difiere de acceso real), se mantiene una inadecuada e insuficiente distribución de los servicios tanto en las zonas rurales como en las urbanas (8, 9), se han denunciado innumerables hechos de corrupción asociados a la apropiación de recursos públicos por parte de las entidades promotoras de salud (EPS), y cada vez son más evidentes las fallas en materia de salud pública. Estos problemas, sumados a los denominados "itinerarios burocráticos" (10) y los procesos administrativos que dilatan la atención (11), han generado problemas serios de acceso que se han hecho especialmente evidentes desde el pronunciamiento de la Corte Constitucional Colombiana y su conocida Sentencia T-760 de 2008.

Dada la situación de déficit en la cantidad, la calidad y la disponibilidad de información sobre acceso a servicios de salud en el país, en el contexto de la crisis estructural de un sistema altamente fragmentado que ocasiona barreras constantemente denunciadas, algunos investigadores colombianos en salud pública han desarrollado proyectos que aportan evidencia significativa, pero que desafortunadamente no han logrado conformar una masa crítica que aporte y promueva cambios de fondo al actual sistema.

Considerando la problemática que se presenta en el sistema y con el propósito de conocer y sistematizar juiciosamente los trabajos de investigación en el tema del acceso a nivel nacional, este estudio tuvo como objetivo presentar una revisión de la literatura que ha abordado el asunto entre los años 2000 y 2013, así como realizar un análisis crítico del estado del arte de la investigación en este campo y de la noción de equidad en el acceso utilizada en algunos de los estudios. Con todo esto se pretende brindar un insumo que sirva para orientar futuras investigaciones que puedan reducir la brecha existente en el estudio de la problemática del acceso a los servicios de salud en Colombia y contribuir a impulsar los cambios que el sistema requiere.

\section{MÉTODO}

En este estudio se realiza una revisión sistemática de los trabajos de investigación en el tema del acceso a los servicios de salud en Colombia. Para este fin se llevó a cabo una búsqueda de artículos publicados entre los años 2000 y 2013 en las bases de datos Ebsco, Jstor, Proquest, ISI Web of Science, Scielo y Cochrane. Para esta búsqueda se establecieron los siguientes descriptores en ingles y español: health services accessibility, health services accessibility and conceptual framework, health services accessibility and theory, health services accessibility and conceptual models, health services accessibility and health systems, equity in access, equity in access to health services, health services accessibility and equity, health services accessibility and survey, measuring health services accessibility, health care access and evaluation, y Colombia.

Como criterio de inclusión se consideró que los artículos fueran estudios empíricos, revisiones sistemáticas o meta-análisis. Como criterio de exclusión se estableció que los estudios no fueran de Colombia, que se tratara de editoriales y artículos de opinión, o 
que hicieran referencia al tema del acceso de manera marginal. A partir de las referencias bibliográficas de la primera búsqueda, se realizó una segunda búsqueda en Google Académico de artículos publicados en la ventana de observación del estudio.

La información extraída de los estudios para efectos de análisis fue: autor (es), año de publicación, ciudad y país, fuentes de financiación de los estudios, tipo de estudio, fuentes de información e instrumentos, modelo teórico de referencia, resultados y conclusión principal. Estos datos fueron sistematizados en una tabla de evidencia que permitiera su posterior categorización y análisis. De esta sistematización surgieron seis categorías principales para el análisis: 1) equidad y acceso a los servicios de salud, 2) efectos de la Ley 100 de 1993 en el acceso a servicios de salud, 3) el acceso en grupos de población y servicios específicos, 4) barreras de acceso a los servicios de salud, 5) fundamentos conceptuales de los estudios sobre acceso a servicios de salud en Colombia y 6 ) variables empleadas en los estudios sobre acceso y fuentes de información.

\section{RESULTADOS}

En total, se recuperaron 71 artículos publicados en el periodo 2000-2013 que contenían los descriptores establecidos para la búsqueda. Teniendo en cuenta los criterios de exclusión y la eliminación de artículos repetidos, este número se redujo a 31 , de los cuales: $64,5 \%$ fueron estudios cuantitativos (20 artículos); $19,35 \%$ cualitativos ( 6 artículos), 9,6\% estudios mixtos (3 artículos), y el 6,45\% restante, revisiones sistemáticas ( 2 artículos). $67,7 \%$ de los estudios se enfocaron en áreas urbanas (21 artículos), 25,8\% analizaron las diferencias entre áreas urbanas y rurales ( 8 16 artículos) y $3,2 \%$ se dirigieron a análisis del acceso en la población rural.
Como se muestra en la tabla $1,90,3 \%$ de los estudios (28 artículos) se realizaron entre los años 2006 y 2011. El 54,8\% de las investigaciones se concentró en ciudades como Bogotá (8 artículos), Medellín (5 artículos), Cali, Pereira, Manizales y Arjona (4 artículos). El 9,67\% efectuó comparaciones entre ciudades ( 3 artículos) y el 9,67\% analizó una región o departamento del país (3 artículos). Una tercera parte de los estudios, $25,8 \%$ (8 artículos) fueron de carácter nacional.

En la tabla 1 también se puede apreciar que, en cuanto a las temáticas de estudio, de los 31 artículos publicados, seis $(19,35 \%)$ se ocuparon del asunto de la equidad en la utilización de servicios, en el tratamiento, en la financiación de la atención, la equidad de etnia o la equidad de género en el acceso. Sobre grupos de población vulnerables, como los desplazados por la violencia y migrantes rurales, se hallaron siete artículos $(22,6 \%)$. Otros siete $(22,6 \%)$ se enfocaron en el acceso a servicios específicos como servicios de salud materno-infantil, urgencias, citología y acciones de promoción y prevención. Por último, diez artículos $(32,2 \%)$ analizaron algunos efectos e implicaciones de la Ley 100 de 1993 en el acceso (se incluyen estudios sobre focalización de subsidios, percepción de los usuarios, comparación entre ciudades y papel de la acción de tutela, entre otros).

De los 31 artículos encontrados, 51,6\% correspondían a investigaciones financiadas exclusivamente por alguna institución universitaria del país (Universidad de Antioquia 6, Pontificia Universidad Javeriana Cali 2, Universidad Nacional de Colombia 2, Universidad del Rosario 2, Universidad del Norte, Universidad Tecnológica de Pereira, Pontificia Universidad Javeriana Bogotá y Universidad del Valle 1). El 12,9\% constituían investigaciones cofinanciadas entre universidades e instituciones gubernamentales o no gubernamentales diferentes a Col- 
ciencias. Otro $12,9 \%$ de los estudios fueron financiados o cofinanciados por Colciencias, 9,7\% por un fondo de organismo internacional y local, 6,5\% por fondos de organismos internacionales, $3,2 \%$ por organizaciones no gubernamentales (ONG) locales y $3,2 \%$ no especifican la fuente de financiación (tabla 1 ).
Hasta aquí, se puede afirmar que en las investigaciones sobre acceso a los servicios de salud en Colombia han predominado los estudios cuantitativos, la mayoría se hicieron durante la segunda mitad de la década del 2000, se enfocaron en áreas urbanas, en las principales ciudades del país (Bogotá, Me-

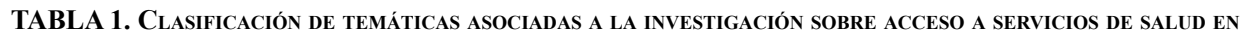
Colombia 2000-2013

\begin{tabular}{|c|c|c|c|c|}
\hline $\begin{array}{l}\text { Temática de } \\
\text { estudio }\end{array}$ & Título del estudio & $\begin{array}{l}\text { Institución financiadora/ } \\
\text { ejecutora del estudio }\end{array}$ & Año & Lugar \\
\hline \multirow{4}{*}{$\begin{array}{l}\text { EQUIDAD EN } \\
\text { EL ACCESO, } \\
\text { UTILIZACIÓN Y } \\
\text { FINANCIACIÓN }\end{array}$} & $\begin{array}{l}\text { Efectos de la reforma de la } \\
\text { seguridad social en salud en } \\
\text { Colombia sobre la equidad en } \\
\text { el acceso y la utilización de } \\
\text { servicios de salud (12) }\end{array}$ & $\begin{array}{l}\text { Programa Equity-Oriented } \\
\text { Health Policy Analysis in } \\
\text { Latin América, de la Red de } \\
\text { Investigación en Sistemas } \\
\text { y Servicios del Cono Sur } \\
\text { (Redinssa) }\end{array}$ & 2000 & Colombia \\
\hline & $\begin{array}{l}\text { Equidad en el acceso a servicios } \\
\text { de salud en Antioquia, Colombia } \\
\text { (13) }\end{array}$ & $\begin{array}{l}\text { Centro de Investigaciones } \\
\text { Económicas de la Facultad } \\
\text { de Ciencias Económicas de la } \\
\text { Universidad de Antioquia }\end{array}$ & 2007 & Antioquia \\
\hline & $\begin{array}{l}\text { Equidad en el acceso a los } \\
\text { servicios de salud y equidad en } \\
\text { la financiación de la atención en } \\
\text { Bogotá (14) }\end{array}$ & $\begin{array}{l}\text { Fondo de Población de las } \\
\text { Naciones Unidas (UNFPA) }\end{array}$ & 2008 & Bogotá \\
\hline & $\begin{array}{l}\text { Estimación de los determinantes } \\
\text { del acceso a los servicios de } \\
\text { salud en la región Caribe (15) }\end{array}$ & $\begin{array}{l}\text { Dirección de Investigaciones y } \\
\text { Proyectos (DIP) de la Universidad } \\
\text { del Norte (Barranquilla, } \\
\text { Colombia) }\end{array}$ & 2008 & Región Caribe \\
\hline $\begin{array}{l}\text { EQUIDAD EN EL } \\
\text { TRATAMIENTO }\end{array}$ & $\begin{array}{l}\text { Equidad en el acceso al } \\
\text { tratamiento para el cáncer de } \\
\text { mama en Colombia (16) }\end{array}$ & $\begin{array}{l}\text { Colciencias y Centro de Estudios } \\
\text { e Investigación en Salud (CEIS) } \\
\text { de la Fundación Santa Fe de } \\
\text { Bogotá }\end{array}$ & 2009 & Estudio nacional \\
\hline $\begin{array}{l}\text { EQUIDAD DE } \\
\text { ETNIA EN EL } \\
\text { ACCESO }\end{array}$ & $\begin{array}{l}\text { Equidad de etnia en el acceso a } \\
\text { los servicios de salud en Bogotá, } \\
\text { Colombia, } 2007 \text { (17) }\end{array}$ & $\begin{array}{l}\text { Universidad Nacional de } \\
\text { Colombia, Sede Bogotá }\end{array}$ & 2008 & Bogotá \\
\hline $\begin{array}{l}\text { EQUIDAD DE } \\
\text { GÉNERO EN EL } \\
\text { ACCESO }\end{array}$ & $\begin{array}{l}\text { Equidad de género en el acceso } \\
\text { a los servicios de salud en } \\
\text { Colombia (18) }\end{array}$ & $\begin{array}{l}\text { Universidad Nacional de } \\
\text { Colombia, Sede Bogotá }\end{array}$ & 2008 & Estudio nacional \\
\hline \multirow{3}{*}{$\begin{array}{l}\text { POBLACIÓN } \\
\text { ESPECÍFICA: } \\
\text { DESPLAZADOS }\end{array}$} & $\begin{array}{l}\text { Vulnerabilidad y exclusión: } \\
\text { Condiciones de vida, situación } \\
\text { de salud y acceso a servicios de } \\
\text { salud de la población desplazada } \\
\text { por la violencia asentada en } \\
\text { Bogotá - Colombia } 2005 \text { (19) }\end{array}$ & $\begin{array}{l}\text { Pontificia Universidad } \\
\text { Javeriana, con financiamiento } \\
\text { del International Development } \\
\text { Research Center (IDRC) de } \\
\text { Canadá y la Secretaría Distrital } \\
\text { de Salud de Bogotá y el apoyo } \\
\text { de la Fundación de Atención al } \\
\text { Migrante (Famig) }\end{array}$ & 2008 & Bogotá \\
\hline & $\begin{array}{l}\text { Factores que inciden en el acceso } \\
\text { de la población desplazada a } \\
\text { las instituciones prestadoras de } \\
\text { servicios de salud en Colombia } \\
(20)\end{array}$ & Universidad del Rosario & 2008 & Bogotá \\
\hline & $\begin{array}{l}\text { Características de la población } \\
\text { desplazada que inciden en el } \\
\text { acceso a los servicios de salud } \\
\text { (21) }\end{array}$ & Universidad del Rosario & 2006 & Bogotá \\
\hline
\end{tabular}




\begin{tabular}{|c|c|c|c|c|}
\hline $\begin{array}{l}\text { Temática de } \\
\text { estudio }\end{array}$ & Título del estudio & $\begin{array}{l}\text { Institución financiadora/ } \\
\text { ejecutora del estudio }\end{array}$ & Año & Lugar \\
\hline & $\begin{array}{l}\text { Apreciaciones de la población } \\
\text { en condición de desplazamiento } \\
\text { forzado sobre los servicios de } \\
\text { salud en algunos municipios de } \\
\text { Colombia (22) }\end{array}$ & $\begin{array}{l}\text { Universidad Tecnológica de } \\
\text { Pereira (Pereira, Colombia) }\end{array}$ & 2009 & $\begin{array}{l}\text { Cali (distrito de } \\
\text { Aguablanca), } \\
\text { Pereira (Villa } \\
\text { Santana)y } \\
\text { Soacha }\end{array}$ \\
\hline & $\begin{array}{l}\text { Barreras de acceso a los } \\
\text { servicios odontológicos de } \\
\text { niños y niñas menores de } 6 \\
\text { años pertenecientes a familias } \\
\text { desplazadas (23) }\end{array}$ & $\begin{array}{l}\text { Facultad de Odontología de la } \\
\text { Universidad de Antioquia }\end{array}$ & 2010 & Medellín \\
\hline & $\begin{array}{l}\text { Caracterización del acceso a los } \\
\text { servicios de salud en población } \\
\text { infantil desplazada y receptora } \\
\text { en asentamientos marginales } \\
\text { en seis ciudades de Colombia, } \\
\text { 2002-2003 (24) }\end{array}$ & Universidad de Antioquia & 2006 & $\begin{array}{l}\text { Cali, Cartagena, } \\
\text { Montería, } \\
\text { Soacha, } \\
\text { Medellín y } \\
\text { Bucaramanga }\end{array}$ \\
\hline $\begin{array}{l}\text { POBLACIÓN } \\
\text { ESPECÍFICA: } \\
\text { MIGRANTES }\end{array}$ & $\begin{array}{l}\text { Accesibilidad al régimen } \\
\text { contributivo de salud en } \\
\text { Colombia: caso de la población } \\
\text { rural migrante }(25)\end{array}$ & $\begin{array}{l}\text { Pontificia Universidad Javeriana- } \\
\text { Cali }\end{array}$ & 2009 & Colombia \\
\hline $\begin{array}{l}\text { SERVICIOS } \\
\text { ESPECÍFICOS: } \\
\text { URGENCIAS }\end{array}$ & $\begin{array}{l}\text { Acceso a los Servicios de } \\
\text { Urgencias en Medellín, } 2006 \\
(26)\end{array}$ & $\begin{array}{l}\text { Instituto Colombiano para } \\
\text { el Desarrollo de la Ciencia y } \\
\text { la Tecnología (Colciencias) } \\
\text { (contrato 313 del 2004) y } \\
\text { Universidad de Antioquia } \\
\text { (Medellín, Colombia), con apoyo } \\
\text { de la Alcaldía de Medellín }\end{array}$ & 2007 & Medellín \\
\hline \multirow{3}{*}{$\begin{array}{l}\text { SERVICIOS } \\
\text { ESPECÍfICOS: } \\
\text { CITOLOGÍA }\end{array}$} & $\begin{array}{l}\text { Accesibilidad a los servicios de } \\
\text { salud en la práctica de citología } \\
\text { reciente de cuello uterino en una } \\
\text { zona urbana de Colombia (27) }\end{array}$ & $\begin{array}{l}\text { División de Salud Fundación } \\
\text { FES-social. }\end{array}$ & 2004 & Bogotá \\
\hline & $\begin{array}{l}\text { La citología de cuello } \\
\text { uterino en Soacha, Colombia: } \\
\text { representaciones sociales, } \\
\text { barreras y motivaciones (28) }\end{array}$ & $\begin{array}{l}\text { Instituto Nacional de } \\
\text { Cancerología, Universidad } \\
\text { Externado de Colombia y } \\
\text { Colciencias }\end{array}$ & 2006 & Bogotá \\
\hline & $\begin{array}{l}\text { Accesibilidad a la citología } \\
\text { cervical en Medellín, Colombia } \\
\text { en } 2006(29)\end{array}$ & $\begin{array}{l}\text { Secretaría de Salud de Medellín } \\
\text { y Universidad de Antioquia }\end{array}$ & 2007 & Medellín \\
\hline \multirow{2}{*}{$\begin{array}{l}\text { SERVICIOS } \\
\text { ESPECÍFICOS: } \\
\text { PROMOCIÓN Y } \\
\text { PREVENCIÓN }\end{array}$} & $\begin{array}{l}\text { Accesibilidad a acciones } \\
\text { de promoción de la salud } \\
\text { y prevención de población } \\
\text { hipertensa de Pereira, Colombia, } \\
\text { 2008. La mirada del paciente } \\
\text { (30) }\end{array}$ & Colciencias & 2009 & Pereira \\
\hline & $\begin{array}{l}\text { Acceso a los servicios } \\
\text { preventivos en los regímenes } \\
\text { contributivo y subsidiado de } \\
\text { salud en un barrio estrato dos de } \\
\text { la ciudad de Cali ( } 31 \text { ) }\end{array}$ & $\begin{array}{l}\text { Vicerrectoría de Investigaciones } \\
\text { de la Universidad del Valle }\end{array}$ & 2011 & Cali \\
\hline $\begin{array}{l}\text { SERVICIO } \\
\text { ESPECÍFICO: } \\
\text { SALUD MATERNA }\end{array}$ & $\begin{array}{l}\text { Determinantes del uso de los } \\
\text { servicios de salud materna en el } \\
\text { litoral Pacífico colombiano (32) }\end{array}$ & $\begin{array}{l}\text { Coordinación Institucional de } \\
\text { Investigaciones de la Pontificia } \\
\text { Universidad Javeriana-Cali }\end{array}$ & & Litoral pacifico \\
\hline $\begin{array}{l}\text { EFECTOS DE } \\
\text { LA LEY } 100 \text { DE } \\
1993 \text { EN EL } \\
\text { ACCESO }\end{array}$ & $\begin{array}{l}\text { Evaluación de los efectos de } \\
\text { la política de focalización de } \\
\text { subsidios en salud sobre la } \\
\text { población sin capacidad de pago } \\
\text { de cuatro localidades de Bogotá, } \\
\text { D. C., Colombia, } 2001 \text { (33) }\end{array}$ & $\begin{array}{l}\text { Fondo Financiero Distrital } \\
\text { de Salud, Hospital del Sur y } \\
\text { Pontificia Universidad Javeriana }\end{array}$ & 2003 & Bogotá \\
\hline
\end{tabular}


Estado del ARTE de LA InVEStigación en ACCESo a los SERVICIOS DE SALUd EN Colombia, 2000-2013: REVISIÓN SISTEMÁTICA CRITICA

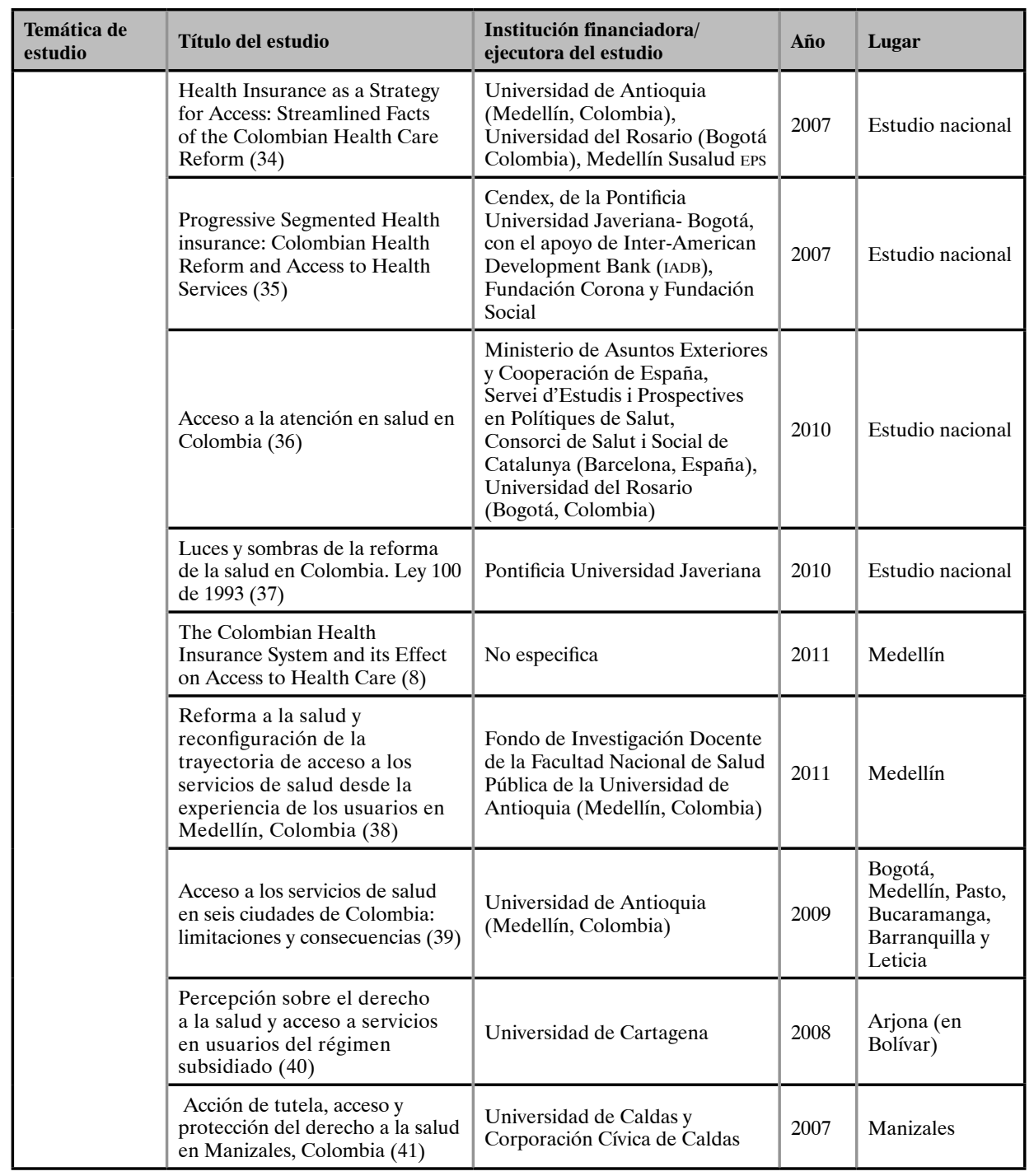

dellín y Cali) y las universidades han sido las principales financiadoras de los estudios. A continuación se presentan los resultados de las categorías de análisis que surgieron de la clasificación de los artículos.

\section{Equidad y acceso a los servicios de salud}

En general, la mayoría de las investigaciones sobre equidad en el acceso a los servicios de salud defienden la tesis de que como resultado de la Ley 100 de 1993 puede considerarse que hay mayor equidad debido a la ampliación de la cobertura, en especial de la población más pobre. Sin embargo, estudios para regiones o ciudades específicas como Bogotá y Antioquia señalan la existencia de inequidades en el acceso a los servicios de salud y a los tratamientos entre la población afiliada y la no afiliada al sistema de salud $(13,16)$; otro estudio enfatiza en las barreras financieras como el principal obstáculo para el acceso de la población más pobre, incluso estando afi- 
liada (14). A nivel nacional, se considera que la población con menor ingreso monetario enfrenta un mayor gasto de bolsillo para la financiación de los servicios de salud, siendo más afectadas las mujeres que los hombres (18). En Bogotá se encontró evidencia de inequidades para el acceso a los servicios de salud derivadas de la pertenencia étnica, en especial entre población negra, que tiene la menor cobertura, a pesar de contar con las mayores necesidades reportadas (17).

En el estudio de las inequidades predominan los estudios cuantitativos y las principales fuentes usadas para su estudio han sido las encuestas de hogares que realiza el Departamento Administrativo Nacional de Estadísticas (DANE) o el Departamento Nacional de Planeación (DNP) como la Encuesta de Calidad de Vida (ECV) de los años 1997 y 2003, la encuesta Nacional de Hogares (ENH) de los años 1994 y 2000 y la Encuesta Nacional de Caracterización Socioeconómica (Casen) de 1993 (12-14, 18). Otros estudios emplean instrumentos ad hoc $(16,17)$.

\section{Efectos de la Ley 100 de 1993 en el acceso a servicios de salud}

Entre las investigaciones que han abordado, en concreto, el efecto de la reforma al sistema de salud sobre el acceso a los servicios, se encuentran dos revisiones sistemáticas. En estas se señalan, por un lado, efectos considerados positivos tales como el aumento en la cobertura, la disminución del gasto de bolsillo, el aumento de la equidad en el aseguramiento mediante la disminución de los diferenciales urbano-rurales, por edad y nivel educativo, la mejor focalización de los subsidios a la población pobre y el aumento del acceso a los servicios de salud entre los asegurados (37). Por otro lado, se presentan limitaciones importantes en los estudios objeto de revisión como la superficialidad de los análisis, con lecturas poco profundas de los resultados; por ejemplo, los estudios no tuvieron en cuenta las diferencias estructurales existentes entre los paquetes de servicios de salud de los regímenes subsidiado y contributivo (37), o no se tuvo en cuenta que estar afiliado como indicador de cobertura, no implica necesariamente tener acceso real a los servicios (36).

Para una mejor comprensión del impacto de la reforma en el acceso a los servicios de salud es preciso que más estudios incorporen la perspectiva de los actores y variables de contexto como las políticas y las características de los proveedores y las aseguradoras (36). Adicionalmente, otras investigaciones se han centrado en el análisis de los determinantes (34) y de las barreras en el acceso $(8,33,34,38)$ que fueron evidentes luego de implementar la reforma. El enfoque predominante en estos estudios ha sido el cualitativo y los estudios se han centrado en territorios específicos. Solo se encontró un estudio con enfoque cuantitativo y alcance nacional (34). Las fuentes de datos que utilizaba fueron las Encuestas de Calidad de Vida de 1997 y de 2003.

\section{El acceso en grupos de población y servicios específicos}

Los estudios para grupos de población específica se han enfocado en la identificación de las barreras de acceso que enfrenta la población desplazada (19-22), incluyendo los niños (23, 24), y en el análisis de los determinantes del acceso al régimen contributivo de la población rural migrante (25). Los estudios sobre el acceso a servicios específicos han analizado el caso de la citología de cuello uterino, las urgencias, servicios de salud materna y los servicios de promoción y prevención. Estos estudios se han enfocado en la caracterización del acceso a los servicios y en la identificación de los determinantes y barreras. Para estas caracterizaciones han predominado los enfoques cuantitativos y 
el empleo de instrumentos ad hoc. Pocos trabajos emplean enfoques cualitativos $(20$, $21,28)$ o encuestas con representatividad nacional y por regiones $(25,32)$.

\section{Barreras de acceso a los servicios de salud}

En general, la mayor parte de las investigaciones reportan barreras de acceso a los servicios de salud y existe consenso entre ellas en que las principales barreras que enfrenta la población colombiana son económicas (34), geográficas, administrativas $(8,12,13,15-17,28,33,38-40)$, culturales $(28,39)$, normativas, de oferta (39), relacionadas con la falta de confianza en el médico (13) y discriminación (17).

Además de los aspectos económicos, las principales barreras que enfrenta la población desplazada para el acceso a los servicios de salud son el bajo nivel educativo y la falta de información sobre aspectos relacionados con el derecho a la salud, requisitos para el uso de servicios médicos y horarios de atención, dificultades para acreditar su condición de desplazado y lograr la cobertura en el Sistema General de Seguridad Social en Salud (sGSss)
$(19,21)$, procedimientos administrativos, capacidad de respuesta de las Empresas Sociales del Estado (ESE) para atender a los desplazados y coordinación entre instituciones para la prestación de los servicios $(19,20,22)$.

\section{Fundamentos conceptuales de los estudios en acceso a servicios de salud en Colombia}

Además de lo anterior, y dada la necesidad de sistematizar y comprender los fundamentos teóricos de los estudios objeto de esta revisión, se identificaron los autores de referencia utilizados, así como la definición del concepto de acceso utilizado. Así, se encontró que 41,9\% (13 artículos) de las investigaciones no exponían la postura teórica en la que se basaban para definir y evaluar el acceso, aunque se podía inferir que era entendido como el uso de servicios de salud o tenencia de seguro de salud $(8,12,18,22$, $25,27,28,32,33,37,39-41)$, mientras que el $58 \%$ (18 artículos) se referenciaron modelos conceptuales predominantes en este campo, como son el modelo de Aday y Andersen $(66,6 \%)$ y el de Frenk $(27,7 \%)$, entre otros. En la tabla 2 se resumen estos resultados.

TABLA 2. Modelos CONCEPTUALES UTILIZAdos EN LA INVESTIGACIÓN SOBRE ACCESO A SERViCIOS DE SALUD. Colombia, 2000-2013

\begin{tabular}{|l|l|l|}
\hline $\begin{array}{l}\text { Autor (es) } \\
\text { de referencia } \\
\text { conceptual }\end{array}$ & Modelo o definición de acceso empleada en el estudio & Estudios \\
\hline $\begin{array}{l}\text { Aday y Ander- } \\
\text { sen (1) }\end{array}$ & $\begin{array}{l}\text { Proponen un modelo de referencia para el estudio del acceso que } \\
\text { parte de la política sanitaria, el cual, a través de las características del } \\
\text { sistema de salud y de la población en riesgo, da como resultados la } \\
\text { utilización de los servicios de salud y la satisfacción del consumidor. }\end{array}$ & $\begin{array}{l}(14-16,21,23, \\
19,30,31, \\
24,38)\end{array}$ \\
\hline $\begin{array}{l}\text { Frenk (4) } \\
\text { Se refiere a la accesibilidad como un proceso (complejo) de búsqueda } \\
\text { y obtención de la atención que depende de la disponibilidad (la } \\
\text { existencia de los recursos para la salud y la capacidad de producción } \\
\text { de servicios de salud) y la utilización de los servicios (determinada por } \\
\text { la resistencia, es decir, los obstáculos de la accesibilidad, reflejados } \\
\text { en costos, ubicación y tiempo en que la persona consigue la cita y es } \\
\text { atendida). }\end{array}$ & $(13,15,19$, & $29,34)$ \\
\hline $\begin{array}{l}\text { Ariza-Monto- } \\
\text { ya y Hernán- } \\
\text { dez-Álvarez } \\
(17)\end{array}$ & $\begin{array}{l}\text { “[..] proceso mediante el cual puede lograrse que una necesidad de } \\
\text { atención, bien sea por el deterioro en el estado de salud, un diagnós- } \\
\text { tico sobre el mismo o la promoción de su mejoramiento, se satisfaga } \\
\text { completamente” (p. 61). }\end{array}$ & $(17)$ \\
\hline
\end{tabular}




\begin{tabular}{|l|l|l|}
\hline $\begin{array}{l}\text { Autor (es) } \\
\text { de referencia } \\
\text { conceptual }\end{array}$ & Modelo o definición de acceso empleada en el estudio & Estudios \\
\hline $\begin{array}{l}\text { Donabedian } \\
(42)\end{array}$ & $\begin{array}{l}\text { La accesibilidad es el "grado de ajuste" entre las características de la } \\
\text { población y las de los recursos de atención de la salud (citado por 15, } \\
\text { p. 108). }\end{array}$ & $(15,29)$ \\
\hline $\begin{array}{l}\text { Ruiz, Amaya y } \\
\text { Venegas (35) }\end{array}$ & $\begin{array}{l}\text { Es la utilización del servicio de salud y su gasto de bolsillo. En el uso se } \\
\text { tienen en cuenta todos los eventos en salud, el uso de servicios ambulato- } \\
\text { rios, de hospitalización y medicamento (p. 9). }\end{array}$ & $(35)$ \\
\hline $\begin{array}{l}\text { Restrepo, } \\
\text { Echeverri, } \\
\text { Vásquez y Ro- } \\
\text { dríguez (43) }\end{array}$ & $\begin{array}{l}\text { "Proceso mediante el cual puede lograrse que una necesidad de } \\
\text { atención, bien sea por el deterioro en el estado de salud, un diagnós- } \\
\text { tico sobre el mismo o la promoción de su mejoramiento, se satisfaga } \\
\text { completamente" (citado por 13, p. 28). }\end{array}$ & $(13)$ \\
\hline $\begin{array}{l}\text { Franco, Ramí- } \\
\text { rez, Escobar, } \\
\text { Isaac y Londo- } \\
\text { ño (23) }\end{array}$ & $\begin{array}{l}\text { El acceso a los servicios de salud es un proceso complejo que involu- } \\
\text { cra la formulación de políticas de atención y hasta la satisfacción del } \\
\text { usuario con el servicio recibido, tomando en cuenta las características } \\
\text { de la población y la organización del servicio para adecuarse a las } \\
\text { necesidades de la población (p. 42). }\end{array}$ & $(23)$ \\
\hline $\begin{array}{l}\text { Valencia, Gon- } \\
\text { zález, Agude- } \\
\text { lo, Acevedo y } \\
\text { Vallejo (26) }\end{array}$ & $\begin{array}{l}\text { El acceso a los servicios de urgencias se entiende como el proceso } \\
\text { mediante el cual se logra conseguir una atención médica inmediata y } \\
\text { oportuna (p. 531). }\end{array}$ & (26) \\
\hline
\end{tabular}

Variables empleadas en los estudios de acceso y fuentes de información

En los estudios revisados se identificaron ocho grupos de variables involucradas con la medición del acceso: i) régimen de aseguramiento (contributivo, subsidiado o no asegurado) (8, $12-16,19,27,28,31,34,35,41)$; ii) variables socioeconómicas $(8,13,16-18,21,24,25$, 27, 30-33, 35); iii) variables demográficas (ubicación geográfica, condiciones laborales, características de la vivienda, pobreza, etnia) ( 12, 13, 15, 16, 17, 19, 24, 27-29, 31, 33, 35); iv) estado de salud percibido (morbilidad y necesidad sentida y percibida) $(13-15,24,31$, 35 ); v) uso del sistema de salud (preventivos, urgencias, curativos, control) $(8,12,14,15$, 19, 21, 22, 27, 30-35, 39); vi) características del sistema de salud (estrategias de atención, información sobre el sistema de salud, exceso de trámites, divulgación de políticas de atención) $(16,20,21)$; vii) capacidad de respuesta institucional (tiempo de espera para la atención y asignación de citas, negación de la atención, resultados en salud, continuidad y calidad de la atención) $(8,16-19,22,24,26,28,37,40)$; viii) características del usuario (morbilidad evaluada, percepción de acceso, condición de vida, satisfacción con el servicio, estrategias para enfrentar las barreras de acceso, fuente de financiación de la atención, predisposición a la consulta, búsqueda de atención) $(13,14$, 18-20, 22-24, 26, 29-31, 35, 38, 40).

Estos indicadores fueron medidos a través de: i) instrumentos construidos ad hoc para ser aplicados en poblaciones específicas (encuestas, formularios, formatos) (10 artículos) $(16,19,22,23,26,27,29-31,40)$, ii) encuestas de hogares con cobertura nacional como la Encuesta de Calidad de Vida (ECV), la Encuesta Nacional de Características Socioeconómica (Casen), la Encuesta Nacional de Hogares (ENH), la Encuesta Continua de Hogares (ECH) o la Encuesta Nacional de Demografía y Salud (ENDS) (10 artículos) $(12-15,17,18,24,25,32,33)$; iii) entrevistas semiestructuradas (5 artículos) $(8,20,21,30$, 41) o en profundidad ( 3 artículos) $(17,33,39)$ y iv) grupos focales (3 artículos) $(17,19,38)$. 


\section{Discusión}

Este estudio tuvo como objetivo conocer y sistematizar los trabajos de investigación en el tema del acceso a servicios de salud en Colombia, mediante una revisión sistemática de literatura de los trabajos realizados entre los años 2000 y 2013, así como realizar un análisis crítico del estado del arte de la investigación en este campo. Los resultados muestran que la mayoría de los estudios se efectuaron entre 2006 y 2011 y analizaron áreas urbanas. Una tercera parte consistió en investigación sobre datos nacionales, las temáticas estuvieron centradas en equidad en la utilización de servicios, grupos de población vulnerables como los desplazados y migrantes rurales, acceso a servicios específicos como servicios de salud materno-infantil, urgencias, citología y acciones de promoción y prevención e implicaciones de la Ley 100 de 1993 en el acceso.

Se encontró, con preocupación, que aproximadamente $42 \%$ de las investigaciones no expusieron la postura teórica en la que se basaron para definir y evaluar el acceso, mientras que $58 \%$ referenciaron modelos conceptuales dominantes en este campo como el de Aday y Andersen $(1,2)$ o el de Frenk (4). En términos metodológicos, predominaron los estudios cuantitativos; los indicadores de acceso fueron medidos o explorados a través de instrumentos construidos ad hoc, encuestas de hogares con cobertura nacional, entrevistas semiestructuradas, entrevistas en profundidad y grupos focales.

A partir de los hallazgos, se pueden discutir como puntos críticos, asuntos relacionados con las perspectivas conceptuales de referencia, la escasez de investigación crítica en el contexto de las sucesivas reformas al sistema luego de la Ley 100 de 1993, las dificultades metodológicas para la investigación en el campo del acceso a servicios de salud y las limitaciones propias de los estudios revisados.

En primer lugar, al revisar los estudios, se encuentra que las perspectivas conceptuales que los fundamentan y el nivel de profundidad en los análisis han sido de diversa naturaleza. Algunos de los estudios han transversalizado el asunto de la equidad en el acceso, pero con divergencias de enfoque. A esto se suma que los enfoques teóricos sobre determinantes del acceso que orientan las investigaciones, a pesar de ser ampliamente utilizados en la literatura, presentan deficiencias al no establecer claramente jerarquías y cadenas de causalidad entre los diferentes factores que componen los modelos. Además, las investigaciones que los usan no han puesto mucho esfuerzo para la adaptación de estos al contexto colombiano, como sí ha ocurrido en otros países (44).

En segundo lugar, el acceso a los servicios de salud y la equidad en el acceso a dichos servicios es un tema de investigación prioritario en Colombia, que veinte años después de la reforma al sistema de salud, se encuentra ante el reto de establecer una nueva reforma que garantice su viabilidad y la solución de problemas estructurales que impiden el acceso equitativo de la población a los servicios de salud y por ende la mejora en los indicadores de salud. En este contexto, se precisan estudios que integren de manera más efectiva para el análisis, no solo los factores que desde el lado de la demanda afectan el acceso, sino también desde la oferta, especialmente en las zonas apartadas y los territorios dispersos del país que es donde se presentan los mayores problemas.

Las investigaciones realizadas hasta el momento han permitido establecer algunas de las barreras y determinantes del acceso a distintos servicios de salud, pruebas diagnósticas y tratamientos. Sin embargo, la 
falta de instrumentos apropiados para la investigación de esta problemática impide extrapolar las conclusiones de muchos de los estudios a todo el territorio nacional, máxime cuando Colombia es un país con regiones tan heterogéneas en términos geográficos, culturales y de desarrollo socioeconómico e institucional.

Los territorios ubicados en la periferia nacional (región de la Orinoquia y Amazonia y Pacífica) donde son mayores las inequidades en el acceso a los diferentes servicios sociales, entre ellos los de salud, han sido en el mejor de los casos poco estudiados, a pesar de albergar a la mayor parte de las minorías étnicas del país y presentar algunos de los peores indicadores de salud y de desarrollo socioeconómico. A esto se suma la presencia de conflicto armado y un medio ambiente hostil (por lo selvático).

En tercer lugar, se evidencian dificultades metodológicas para la investigación en el campo del acceso a servicios de salud, principalmente, la falta de fuentes de información apropiadas. Por ejemplo, las investigaciones sobre el acceso a los servicios de salud con muestras representativas a nivel nacional y de las diferentes regiones y territorios del país han estado limitadas por la disponibilidad de información de las encuestas de hogares, cuyos módulos sobre salud son insuficientes para un estudio profundo de esta problemática. Si bien estas encuestas recogen información profunda sobre las condiciones sociales y económicas de la población, los módulos sobre salud son limitados y generalmente recogen información referente a la cobertura en salud, a las fuentes de financiación del gasto en salud, el autorreporte del estado de salud y la decisión de consultar al médico, entre otras variables. En otros casos, como los de la Encuesta Nacional de Demografía y Salud, la información sobre salud es abundante pero está enfocada en un grupo de población específica y los módulos de información socioeconómica son más pequeños. Además, las encuestas tienen poca información sobre aspectos relacionados con la oferta de los servicios.

Finalmente, hacen falta estudios que aborden de manera integral todos los eslabones que involucran el acceso a los servicios de salud. Generalmente, se estudia el acceso en un momento específico del proceso (consulta inicial, búsqueda de tratamiento, etc.), pero son muy pocos los estudios que han intentado seguir el proceso completo desde que se requiere el servicio hasta que el problema de salud es resuelto. La mayoría de estudios se han centrado en el acceso desde el punto de vista del uso del servicio de salud, especialmente la cita o los exámenes diagnósticos, pero solo se encontró un estudio sobre el tratamiento.

\section{Referencias bibliográficas}

1. Aday LA, Andersen R. A Framework for the Study of Access to Medical Care. Health Services Research. 1974; 9 (3): 208-20.

2. Andersen RM. Revisiting the Behavioral Model and Access to Medical Care: Does it Matter? Journal of Health and Social Behavior. 1995; 36: 1-10.

3. Penchansky R, Thomas WJ. The Concept of Access: Definition and Relationship to Consumer Satisfaction. Med Care. 1981; 19: 127-40.

4. Frenk J. El concepto y la medición de accesibilidad. Salud Pública de México. 1985; 27 (5): 438-53.

5. Levesque JF, Harris M, Russell G. Patient-Centred Access to Health Care: Conceptualizing Access at the Interface of Health Systems and Populations. International Journal for Equity in Health. 2013; 12:18. DOI:10.1186/1475-9276-12-18

6. Colombia, Ministerio de Salud. Estadísticas prestación de servicios: Cuadro de indicadores de prestación [Internet] [acceso: 3 de septiembre del 2012]. Disponible en: http://www.minsalud.gov.co/ estadisticas/default.aspx

7. Colombia, Ministerio de Salud. Estadísticas de aseguramiento. Base de datos unificada de afiliados [Internet] [acceso: 17 de mayo del 2014]. Disponible en: http://www.minsalud.gov.co/estadisticas/ default.aspx

8. Alvarez LS, Salmon JW, Swartzman D. The Colombian Health Insurance System and its Effect on Access to Health Care. Int J Health Serv. 2011; 41 (2): 355-70. 
9. Vargas-Lorenzo I, Vázquez-Navarrete ML, MogollónPérez AS. Acceso a la atención en salud en Colombia. Rev Salud Pública. 2010; 12 (5): 701-12.

10. Abadía-Barrero CE, Oviedo-Manrique DG. Bureaucratic Itineraries in Colombia. A Theoretical and Methodological Tool to Assess Managed-Care Health Care Systems. Social Science \& Medicine. 2009; 68 (6): 1153-60.

11. Observatorio de la Seguridad Social. Aspectos económicos de la reforma a la salud en Colombia. 2013; 12 (26): 1-31.

12. Céspedes JE, Jaramillo I, Martínez R, Olaya S, Reynales J, Uribe C et ál. Efectos de la reforma de la seguridad social en salud en Colombia sobre la equidad en el acceso y la utilización de servicios de salud. Rev Salud Pública. 2000; 2 (2): 145-64.

13. Mejía-Mejía A, Sánchez-Gandur AF, TamayoRamírez JC. Equidad en el acceso a servicios de salud en Antioquia, Colombia. Revista de Salud Pública. 2007; 9 (1): 26-33.

14. Rubio-Mendoza ML. Equidad en el acceso a los servicios de salud y equidad en la financiación de la atención en Bogotá. Rev Salud Pública. 2008; 10 sup (1): 29-43.

15. Rodríguez S, Roldán P. Estimación de los determinantes del acceso a los servicios de salud en la Región Caribe. Revista Economía del Caribe. 2008; 2: 110-12.

16. Velásquez-de Charry LC, Carrasquilla G, RocaGaravito S. Equidad en el acceso al tratamiento para el cáncer de mama en Colombia. Salud Pública Mex. 2009; 51 Supl 2: s246-s253.

17. Ariza-Montoya JF, Hernández-Álvarez ME. Equidad de etnia en el acceso a los servicios de salud en Bogotá, Colombia, 2007. Rev Salud Pública. 2008; 10 Sup (1): 58-71.

18. Guarnizo-Herreño CC, Agudelo C. Equidad de género en el acceso a los servicios de salud en Colombia. Rev Salud Pública. 2008; 10 Sup (1): 44-57.

19. Hernández-Bello A, Gutiérrez-Bonilla ML. Vulnerabilidad y exclusión: Condiciones de vida, situación de salud y acceso a servicios de salud de la población desplazada por la violencia asentada en Bogotá-Colombia, 2005. Rev Gerenc Polit Salud. 2008; 7 (14): 145-76.

20. Mogollón-Pérez AS, Vázquez ML. Factores que inciden en el acceso de la población desplazada a las instituciones prestadoras de servicios de salud en Colombia. Cad. Saúde Pública. 2008; 24 (4): 745-54.

21. Mogollón-Pérez AS, Vázquez-Navarrete ML. Características de la población desplazada que inciden en el acceso a los servicios de salud. Rev Gerenc Polit Salud. 2006; 5 (11): 96-110.

22. Moreno GA, Monsalve JC, Tabima D, Escobar JI. Apreciaciones de la población en condición de desplazamiento forzado sobre los servicios de salud en algunos municipios de Colombia. Rev Fac Nac Sal Púb. 2009; 27 (2): 131-41.

23. Franco AM, Ramírez S, Escobar G, Isaac M, Londoño PA. Barreras de acceso a los servicios odontológicos de niños y niñas menores de 6 años pertenecientes a familias desplazadas. Rev CES Odont. 2010; 23 (2): 41-8.
24. Ballesteros MP, Gaviria MB, Martínez SE. Caracterización del acceso a los servicios de salud en población infantil desplazada y receptora en asentamientos marginales en seis ciudades de Colombia, 2002-2003. Rev Fac Nac Sal Púb. 2006; 24 (1): 7-17.

25. Arroyo S, Tovar LM. Accesibilidad al régimen contributivo de salud en Colombia: caso de la población rural migrante. Desarrollo y Sociedad. 2009; 64: 153-72.

26. Valencia ML, González G, Agudelo NA, Acevedo L, Vallejo IC. Acceso a los servicios de urgencias en Medellín, 2006. Rev Sal Púb. 2007; 9 (4): 529-40.

27. Lucumí-Cuesta DI, Gómez Gutiérrez LF. Accesibilidad a los servicios de salud en la práctica de citología reciente de cuello uterino en una zona urbana de Colombia. Rev Esp Sal Púb. 2004; 78: 367-77.

28. Wiesner-Ceballos C, Vejarano-Velandia M, Caicedo-Mera JC, Tovar-Murillo SL, Cendales-Duarte R. La citología de cuello uterino en Soacha, Colombia: representaciones sociales, barreras y motivaciones. Rev Sal Púb. 2006; 8 (3): 185-96.

29. Restrepo J, Mejía A, Valencia M, Tamayo L, Salas W. Accesibilidad a la citología cervical en Medellín, Colombia en 2006. Rev Esp Sal Púb. 2007; 81 (6): 657-66.

30. Muñoz L, Cerezo M, González M, Paz A, Realpe C, Vélez Cet ál. Accesibilidad a acciones de promoción de la salud y prevención de población hipertensa de Pereira, Colombia, 2008. La mirada del paciente. Investigaciones Andina. 2009; 18 (11): 6-21.

31. Díaz-Grajales C, Zapata-Bermúdez Y, AristizábalGrisales JC. Acceso a los servicios preventivos en los regímenes contributivo y subsidiado de salud en un barrio estrato dos de la ciudad de Cali. Rev Gerenc Polit Sal. 2011; 10 (21): 153-75.

32. Aguado-Quintero LF, Girón-Cruz LE, Osorio Mejía AM, Tovar Cuevas LM, Ahumada-Castro JR. Determinantes del uso de los servicios de salud materna en el litoral Pacífico colombiano. Rev Latinoam Cienc Soc Niñez Juv. 2007 ; 5 (1): 1-36.

33. Vega RR et ál. Evaluación de los efectos de la política de focalización de subsidios en salud sobre la población sin capacidad de pago de cuatro localidades de Bogotá, D. C., Colombia, 2001. Rev Sal Púb. 2003, 5 (1): 59-86.

34. Restrepo J, Zambrano A, Velez M, Ramírez M. Health Insurance as a Strategy for Access: Streamlined Facts of the Colombian Health Care Reform [documento de trabajo]. Bogotá: Universidad del Rosario; 2007.

35. Ruíz F, Amaya L, Venegas S. Progressive Segmented Health Insurance: Colombian Health Reform and Access to Health Services. Health Econ. 2007; 16: 3-18.

36. Vargas-Lorenzo I, Vázquez-Navarrete ML, Mogollón-Pérez AS. Acceso a la atención en salud en Colombia Rev Sal Púb. 2010; 12 (5): 701-12.

37. Yepes-Luján FJ. Luces y sombras de la reforma de la salud en Colombia. Ley 100 de 1993. Rev Gerenc Polít Sal. 2010; 9 (18): 118-23.

38. Echeverry-López ME. Reforma a la salud y reconfiguración de la trayectoria de acceso a los servicios de salud desde la experiencia de los usuarios en Medellín Colombia. Rev Gerenc Polit Sal. 2011; 10 (20): 97-109. 
39. Vargas J, Molina G. Acceso a los servicios de salud en seis ciudades de Colombia: limitaciones y consecuencias. Rev Fac Nac Sal Púb. 2009; 27 (2): 121-30.

40. Alvis-Guzmán N, Alvis-Estrada L, Orozco-Africano J. Percepción sobre el derecho a la salud y acceso a servicios en usuarios del régimen subsidiado en un municipio colombiano, 2005. Rev Sal Púb. 2008; 10 (3): 386-94.

41. Vélez-Arango AL, Realpe-Delgado C, GonzagaValencia J, Castro-Castro AP. Acción de tutela, acceso y protección del derecho a la salud en Manizales, Colombia. Rev Sal Púb. 2007; 9 (2): 297-30.
42. Donabedian A. Aspects of Medical Care Administration. Cambridge, Estados Unidos: Harvard University Press; 1973.

43. Restrepo J, Echeverri E, Vásquez J, Rodríguez S. El seguro subsidiado y el acceso a los servicios de salud. Teoría, contexto colombiano y experiencia en Antioquia. Medellín: Centro de Investigaciones Económicas, Universidad de Antioquia; 2006.

44. Tamez-González S, Valle-Arcos RI, Eibenschutzhartman C, Méndez-Ramírez I. Adaptación del modelo de Andersen al contexto mexicano: acceso a la atención prenatal. Sal Púb Méx. 2006; 48 (5): 418-29. 